\title{
Opinion
}

\section{Darwin and Huxley revisited: the origin of allometry Charles F Stevens}

Address: The Salk Institute, 10010 North Torrey Pines Road, La Jolla, CA 92037, USA. Email: stevens@salk.edu

Published: 23 February 2009

Journal of Biology 2009, 8:14 (doi: 10.1 186/jbioll 19)

The electronic version of this article is the complete one and can be found online at http://jbiol.com/content/8/2/I4

(C) 2009 BioMed Central Ltd

\begin{abstract}
The relative sizes of parts of an organism frequently depend on the absolute size of the individual, a relationship that is generally described by power laws. I show here that these power laws are a consequence of the way evolution operates.
\end{abstract}

The Huxley whose name appears in the title of this article is not Thomas (Darwin's 'Bulldog'), but his grandson Julian, the author of Problems of Relative Growth [1], brother of the novelist Aldous (Brave New World) and half-brother of the biophysicist Andrew (the Hodgkin-Huxley equations). In 1924, Julian began studying the relative size of various organs and found many examples of what he called 'allometries', as opposed to 'isometries'. If an individual were simply magnified, all the parts would increase in size by the same amount and this would be an example of an isometry, in which the relative size of the component parts is independent of the absolute size of the organism. But the heads of the ants in Figure 1a are relatively larger for a large ant than for a small one, and this is an example of an allometric relationship: the relative size of one part of an organism compared to another part depends on the absolute size of the individual.

The remarkable thing Huxley discovered in his studies of relative growth, and summarized in his 1932 book Problems of Relative Growth [1], is that the mathematical relationship describing an allometry is very often a power law rather than some other function such as an exponential or a sigmoidal curve. From Figure 1a one can see that the ant legs, thorax and abdomen are about isometric (close to the same relative size for all the ants pictured), but the length of the head, $L$, is related to the length of the abdomen, $A$, by the power law $L=b A^{k}$, with $k=2.06$. The width of the head, $W$, is related to $A$ by another power law, $W=b A^{k}$, with the exponent $k=1.66$. When power laws are plotted double logarithmically, they give straight lines with a slope of $k$ (Figure 1b). It is easy to imagine that these double logarithmic plots would be curved rather than straight (not power laws), but in fact they typically are close to straight lines, sometimes over a 1,000-fold or more range of sizes. Allometric relationships like these are also called 'scaling laws' in the broader context of the physical sciences.

Why should power laws pop up so frequently in studies of the relative sizes of parts of an organism? A common view is that their frequent appearance is an illusion. Problems of Relative Growth was an immediate success, but Huxley's argument that allometric relationships are described by power laws was viewed with suspicion from the very start. For example, in his review of Huxley's book in Nature, CFA Pantin [2] concluded that "It is a book which every biological library should possess and every student of biology can read with profit." Nevertheless, Pantin noted that the power-law relation "... is necessarily empirical. Of the causes of differential growth we have little knowledge; their investigation is the problem at issue. A variety of possible relations, in fact, reduce approximately to this formula. But it is not the object of the formula to establish the correctness of a particular hypothesis as the cause of differential growth; it merely expresses the observed facts with considerable accuracy in a simple way, so that many very significant features emerge which would not otherwise do so." 


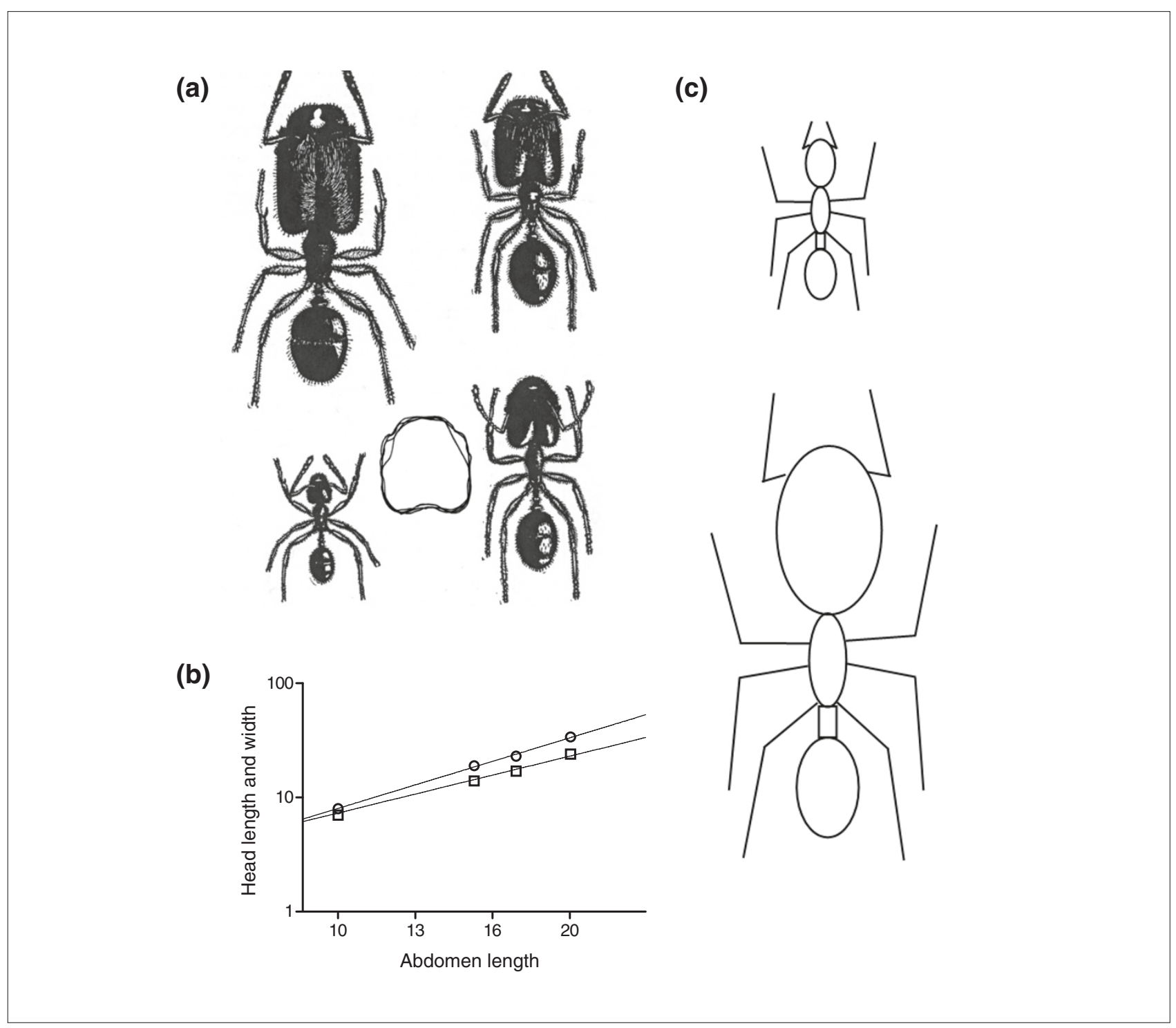

Figure I

Allometric relations are illustrated by pictures and graphs. (a) Four neuters of the ant Pheidole instabilis reproduced from Figure 36 of Huxley's Problems of Relative Growth [1]. The size of the head is clearly relatively larger for the larger ants, an example of an allometric relationship. The line drawing between the two smallest ants shows the outlines of the four ant heads superimposed, after being scaled in the $x$ and $y$ directions to be the same size. (b) A double logarithmic plot of head length (circles) and head width (squares) as a function of abdomen length (arbitrary units) for the four ants in (a). (c) Idealized ants with elliptical heads illustrate an allometric relationship between heads and bodies.

Pantin's early reserve about the use of power laws has continued. For example, in his comprehensive review of allometry, Gould [3] said that allometry "... is not confined to any form of mathematical expression, such as a power function."

Here I show, however, that Huxley's power laws are not just a convenience but rather often are a natural consequence of the way evolution operates.

\section{The state of evolutionary theory on Darwin's 100th} birthday

Julian Huxley graduated from Oxford just 100 years after the birth of Charles Darwin, and after attending the centennial celebrations, embarked on a career in biology. Later in his career, Huxley was an important participant in the incorporation of population genetics into Darwinian theory - the synthetic or neo-Darwinian theory of evolution but his first major contribution was the study of allometric 
relations. To understand why Huxley was so attracted to scaling laws in biology, I first need to briefly review the state of evolutionary theory in 1909 [4].

By the start of the 20th century, virtually all biologists embraced the notion of evolution. But at that time there was a sharp debate about the mechanism of evolutionary change. As Huxley [5] wrote later, in a review of the opposition to Darwin's theory at the turn of the century, "... about 1890 doubts began to be thrown upon it, and around 1910 it had become so unfashionable that some critics proclaimed the death of Darwinism." 'Darwinism' here refers to the random heritable variation and natural selection parts of the theory, not the idea of evolution itself. One of the popular alternatives to natural selection as a mechanism for evolution was the notion of 'orthogenesis' [6]. The 19thcentury concept of orthogenesis is a little difficult to grasp by the 21st-century mind used to the biochemical mechanisms of genetics because the mode of explanation common around 1900 is so unfamiliar to us. Orthogenesis is synonymous with 'definitely directed evolution' [7], the idea that organisms contain a principle that causes them to transmutate (in part, perhaps, through some stimulus from the environment) along a predetermined course. The course of this evolution is unaffected by natural selection unless the result is so badly suited to the environment that the species becomes extinct. This theory was particularly appealing to paleontologists, whose arrangement of fossils in an 'orthogenetic series' revealed what was believed to be the natural unfolding of a predetermined program of change such as, for example, a steady increase in size. To explain the appeal of orthogenesis, I need to consider the 19thcentury criticisms of Darwin.

Of the many early criticisms of Darwinian theory [4] three are important here. First, critics noted that not all changes between species could be believed to be adaptive; indeed, arguments for the adaptive nature of most characteristics of a species were really 'Just So' stories [8]. Second, the critics had a hard time accepting Darwin's notion that changes occurred totally at random, because various species seemed to develop along some orderly trajectory as if evolution had a preferred direction. Also, by the time of Darwin's 100th birthday, the theory of recapitulation ('ontogeny recapitulates phylogeny') had many adherents and, because development follows a predetermined course, how could evolution be directionless? Finally, biologists at the time hoped that evolution would conform to laws (orthogenesis was claimed, by its proponents, to be a law), and the concept of a probabilistic law that Darwinism demanded was largely unfamiliar, certainly in biology. The law of orthogenesis, then, was preferred by many over the natural selection of random changes as a mechanism for evolution because it escaped (by construction) these three criticisms. Some versions of orthogenesis also accounted for the extinction of species because it was believed that, in the final stages of the predetermined evolutionary changes, maladaptive characteristics, like the giant antlers thought to cause the extinction of the Irish elk, were a natural end state.

In 1924 Huxley published his first paper [9] on allometry (he used the term 'hetergonic development' at the time and did not coin 'allometry' until 1936 [10]) in which he analyzed data on the relative size of the larger fiddler crab claw (chela; Figure 2a). When the weight of the large claw of the fiddler crab was plotted double logarithmically against the weight of the rest of the body, "... a remarkably straight line was obtained" (Figure 2b). This finding is significant, according to Huxley, because of its implications for evolutionary theory. If these allometric relationships hold (as Huxley found they did) "... throughout a group, and the evolution of the group has been from small to large size, we shall get apparent orthogenesis..." [9]. Thus, the existence of allometric relationships, together with natural selection for size, automatically explains orthogenesis within the context of Darwinian theory. This is Huxley's important observation but, of course, it leaves open the question of why allometric relations exist at all.

\section{The state of evolutionary theory on Darwin's 200th birthday}

By Darwin's 150th birthday, the original evolutionary theory had been updated - Huxley was an important contributor to this updating - to neo-Darwinism, a version that incorporated population genetics to give a mechanistic account of the sources of variability upon which natural selection worked. Now, at Darwin's 200th birthday, evolutionary theory reflects the inclusion of the revolution in molecular biology that occurred during the last half of the 20th century and into this one [11-13].

Orthogenesis was constructed as an answer to particular criticisms of Darwin's theory, and these criticisms, although still relevant, tended to be obscured by the middle of the 20th century by the success of neo-Darwinism in removing the mystery associated with 'heritable variations'. Nevertheless, species do evolve in preferred directions and do possess many individual characteristics that have not arisen as a direct result of selection. With the re-merging of development and evolution [11-13], we now appreciate that pattern formation in embryogenesis is the result of complex genetic networks, and that these networks are highly conserved through evolution. The constraints imposed by the patternformation networks account for the preferred directions of evolution and the existence of characteristics that are not 
(a)

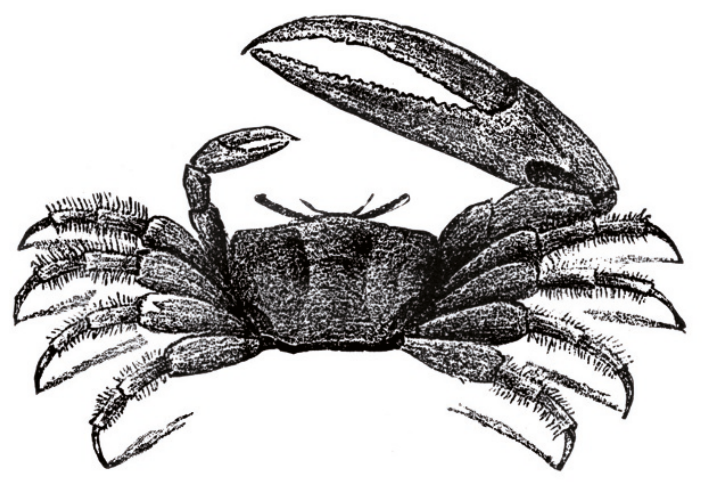

(b)

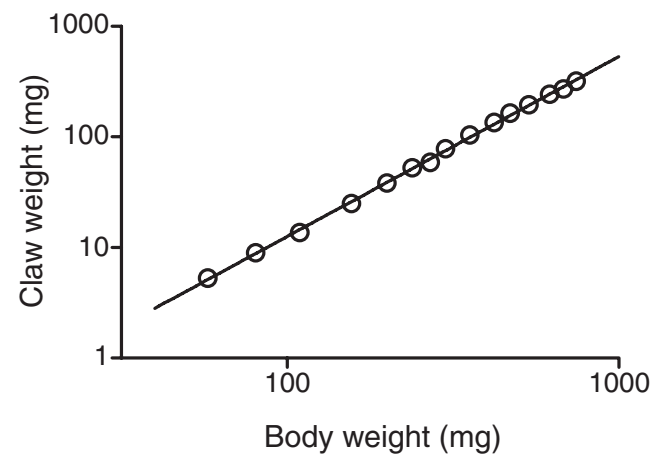

Figure 2

(a) Fiddler crab. Clipart courtesy of Florida Center for Instuctional Technology [http://etc.usf.edu/clipart]. (b) Double logarithmic plot of Huxley's original data upon which his first paper [9] was based, with Uca pugnax claw weight on the ordinate and the crab weight (minus the claw weight) on the abscissa. Data reported in Table I a of [I] for sexually immature male crabs.

necessarily adaptive, and explain why the size of organs is related to the overall body size. Thus, the existence of allometric relations has, for the past two decades, been attributed to conservation of genetic networks [14].

\section{How power laws arise}

But why do we so often seem to end up with power laws when we describe the relative size of organs? Here is my answer to this question.

Figure 1a shows drawings of ants reproduced from Figure 36 of Huxley's Problems of Relative Growth [1]. The line drawing inserted between the smallest two ants represents the superimposed outlines of heads for all four ants after the outlines have been stretched or compressed in the vertical and horizontal directions to be all the same width and length. Clearly, the ant heads are all about the same shape, but stretched and compressed by different amounts. This observation is, as described below, what leads to the allometric relationships presented in Figure 1b (log of head length and width as a function of log abdomen length).

The outline drawings of the ant heads in Figure 1a can be described by some, perhaps complicated, function of $x$ (horizontal direction) and $y$ (vertical direction). A single functional form can be used to describe approximately all four heads because they all have the same shape except they are stretched and shrunk in the $x$ and $y$ directions. Because the function that describes the head shape is not a familiar and simple one, I will make the following arguments assuming that the head shape is an ellipse. The real head shape is not, of course, but the argument I make holds no matter what function is used, and ellipses provide an easy example. Also, I should describe the idealized ant head by a three-dimensional ellipsoid, but the two-dimensional case is simpler and the arguments are just the same for three dimensions.

So, two of our idealized ants are represented in Figure 1c with their heads described by the equation for an ellipse:

$$
\left(\frac{x}{W(s)}\right)^{2}+\left(\frac{y}{L(s)}\right)^{2}=1,
$$

where $x$ is the horizontal coordinate, $y$ is the vertical coordinate, $s$ is the abdomen length, and $L(s)$ and $W(s)$ determine the length and width of the head ( $L$ for the vertical axis of the ellipse and $W$ for the horizontal axis). Note that the values of $L(s)$ and $W(s)$ depend on the size $s$; when $L(s)$ is increased or decreased, the ellipse is stretched or compressed along the vertical axis, with $W(s)$ controlling the ellipse size along the $x$ axis in the same way. For the idealized small ant in Figure $1 c, s=1, L(1)=1$, and $W(1)=0.6$, and for the large ant, $s=2, L(2)=3.3$, and $W(2)=2$.

How do $W(s)$ and $L(s)$, the head length and width, depend on $s$, a measure of body size? Generally, we would expect both length and width to increase smoothly (continuously) with body size. For example, Darwin's finches have beaks 


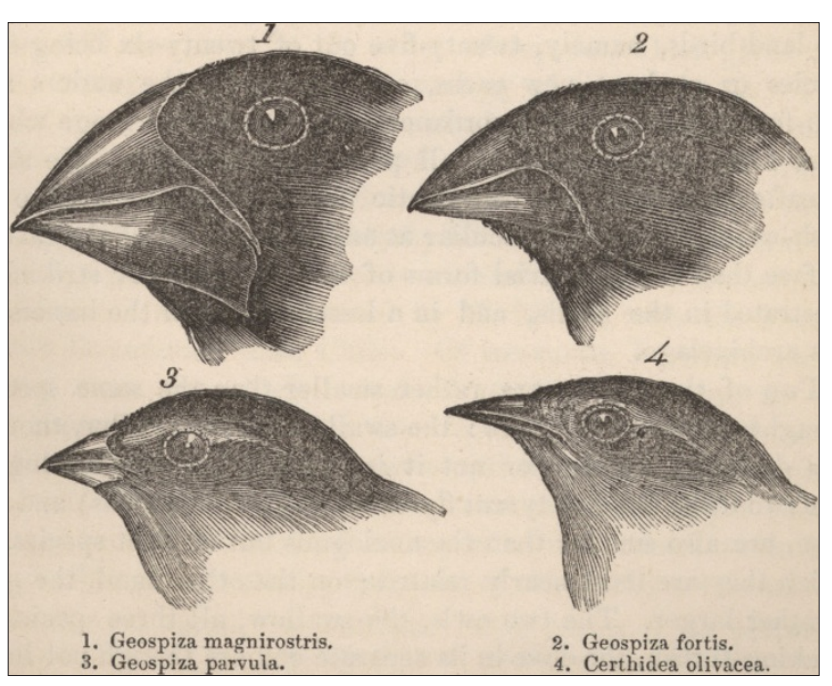

Figure 3

Darwin's or Galapagos finches. From Darwin's account of his voyage on HMS Beagle [23].

whose length depends (monotonically) on the calmodulin concentration, and whose width depends (monotonically) on expression of the developmental signal protein BMP4 during development [15,16] (Figure 3). (Note that size $s$ above might not necessarily be measured by the size of an organ, but by the concentration of some signaling molecule or the expression level of a transcription factor during a particular period of the individual's life.)

The essential feature illustrated in Figure $1 \mathrm{c}$ is that a single equation describes both ant heads, and this equation contains parameters (here $L$ and $W$ ) that depend on some size factor (here, abdomen length) and determines the head size of the ant. Equations of this sort have been known for a long time and are called 'homogeneous' or 'self-similar'. Selfsimilar equations have a remarkable property $[17,18]$ : the $L(s)$ and $W(s)$ can only be power functions (see Appendix), such as:

$$
L(s)=b s^{k}
$$

where $b$ and $k$ are constants. This means that whenever evolution has conserved the genetic networks responsible for pattern formation so that structures are, from one individual to the next, very nearly the same shape (up to stretching and compression), Huxley's power-law relationships must arise. Power laws do not just happen to work sometimes - they are required whenever evolutionary mechanisms preserve organ shapes (except for stretching or shrinking in one or another direction).

\section{Two challenges for contemporary allometry}

Stephen Jay Gould [3] stressed the distinction between allometric relations that occur within one species (like the fiddler crab example) and those that occur across species (for example, brain size versus body size for various primates [19]). I have not honored this distinction above, because my argument holds for both cases, and because the question for any allometric relation ultimately is: how was the scaling relation generated in embryogenesis? One challenge in understanding allometry, then, is finding an answer to this question. Presumably, any conserved patternformation mechanism has been selected because it permitted the existence of allometric relations so that one mechanism would work for an individual of any size.

But even if the developmental mechanisms that produced a particular allometric relation were known, there is still the question of why evolution selected that particular allometry. The allometric relation must serve a purpose, so what is it? Questions about allometric relationships fall, then, into two categories: How is the allometry generated and what is it good for?

Explanations for the advantages of a particular allometric relation often boil down to accounting for the value of the exponent in the power law ( $k$ in the equations above). Whenever we believe an allometry is indeed described by a power law, it should be possible to attempt an explanation for why the exponent in the power law has the particular value it has. For example, the value of the exponent in Kleiber's law (the 3/4 power-law relationship between the mass of an organism and its metabolic rate) has been accounted for by properties of transport in fractal distribution systems [20]. In another example, the $3 / 2$ power law relating the number of neurons that provide the primate visual cortex with information to the number of neurons that process that information is claimed to arise because the primary visual cortex increases the dimensionality of the neural representation of the visual world from two to three [21].

Another type of study derives from the mere existence of an allomeric relation. If evolution is going to work on the brain, the computational power of neural circuits must be scaled to meet the demands placed on them. For example, a visual system in primates of various sizes must be able to process the information from retinas with different numbers of photoreceptors, and the circuits that do this cannot be completely redesigned every time the number of photoreceptors is changed. What this means is that the neural circuits in the brain must have what computer scientists call a scalable architecture, a circuit design whose computational power can be increased by making the circuit larger. This requirement of scalability places strong 
constraints on the types of computations that can be carried out by brain regions [22], and an important challenge resulting from allometry is to learn the design principles that are used in building a brain with a scalable architecture.

The preceding discussion has focused on the logic of allometric relations, but probably the greatest challenge is understanding their developmental origin. Embryonic growth occurs in a way that the correct size relations between all organs result, so there must be general mechanisms of development that govern organ size, and these mechanisms must be remarkably robust. One of the great challenges, then, is to discover how these size relations are generated.

\section{Appendix}

The purpose of this appendix is to show how power laws arise inevitably in the context of self-similar functions, those functions that describe the same shape except for stretching and compression along two orthogonal axes. The equation for an ellipse given above,

$$
\left(\frac{x}{W(s)}\right)^{2}+\left(\frac{y}{L(s)}\right)^{2}=1 \text {, }
$$

can be solved for $y$ to give

$$
y=L(s) \sqrt{1-\left(\frac{x}{W(s)}\right)^{2}}
$$

and this has the form

$$
y=f(x, s)=\alpha(s) f(\beta(s) x, 1)
$$

where $\alpha(s)=L(s), \beta(s)=1 / W(s)$, and $f(x, 1)=\sqrt{ }\left(1-x^{2}\right)$. Any function $g(x, s t)$ that has the form

$$
g(x, s t)=\alpha(s) g(\beta(s) x, t)
$$

is self-similar [18] so that the ellipse is a particular selfsimilar function with $t=1$ and $g=f$. Self-similar functions are so called because the graph for any value of $s$ can be made to superimpose on the graph with $s=1$ by stretching or shrinking along the $x$ and $y$ axes. The remarkable result is that $\alpha(s)$ and $\beta(s)$ must be power functions if they depend continuously on the size parameter $s$ [17]. I have considered only two-dimensional functions here, but the arguments are the same for three-dimensional functions. What this means is that allometric relationships must result if organs for different individuals are the same shape (up to stretching and shrinking) and depend continuously on a size parameter. The goal now is to show that $\alpha(s)$ and $\beta(s)$ are, indeed, power functions. I make the reasonable assumption that these functions are differentiable, but weaker requirements give the same answer [17].
From the definition of a self-similar function, I can write

$$
g(x, s t)=\alpha(s) g(\beta(s) x, t)=\alpha(s) \alpha(t) g(\beta(s) \beta(t) x, 1),
$$

but it is also true (again by the definition) that

$$
g(x, s t)=\alpha(s t) g(\beta(s t) x, 1)
$$

This pair of equations means that we get the functional equations:

$$
\alpha(s t)=\alpha(s) \alpha(t) \text { and } \beta(s t)=\beta(s) \beta(t)
$$

Note that $\alpha(1)=1$ (and $\beta(1)=1)$ because, when $t=1$, we find that $\alpha(s)=\alpha(1) \alpha(s)$.

To solve a functional equation of this sort, I now take the derivative with respect to $t$ (using the chain rule) of the equation for $\alpha(s t)$, and then set $t=1$. The result is

$$
s \frac{d \alpha(s)}{d s}=\alpha^{\prime}(1) \alpha(s)
$$

which can be rearranged to give

$$
\frac{d \alpha(s)}{\alpha(s)}=\alpha^{\prime} \frac{d s}{s}
$$

and this equation can be integrated to give

$$
\log (\alpha(s))=k \log (s)
$$

where I have defined $k \equiv \alpha(1)$ and made use of the fact that $\alpha(1)=1$. Thus, $\alpha(s)$ is the power law

$$
\alpha(s)=s^{k},
$$

with the same result for $\beta(s)$ except that the value of the exponent can be different. The prefactor $b$ in the power laws that appear in the main text would be found from $g(0,1)$ for the axis in the $y$ direction and from solving $g(x, 1)=0$ for the axis in the $x$ direction because I have chosen the origin of the coordinate system to fall at the center of the plane curve described by the self-similar function $g$. 


\section{References}

I. Huxley JS: Problems of Relative Growth. [reprint] Baltimore: Johns Hopkins University Press; 1993.

2. Pantin CFA: Form and size. Nature 1932, 129:775-777.

3. Gould SJ: Allometry and size in ontogeny and phylogeny. Biol Rev Camb Philos Soc 1966, 41:587-640.

4. Bowler PJ: The Eclipse of Darwinism. Baltimore: Johns Hopkins University Press; 1983.

5. Huxley JS: Man in the Modern World. New York: New American Library; 1948.

6. Bowler PJ: Theodor Eimer and orthogenesis: evolution by 'definitely directed variation'. J Hist Med Allied Sci 1979, 34:40-73.

7. Eimer T: On Orthogenesis and the Impotence of Natural Selection in Species-formation. Chicago: The Open Court Publishing Company; 1897.

8. Gould SJ, Lewontin RC: The spandrels of San Marco and the Panglossian paradigm: a critique of the adaptationist programme. Proc $R$ Soc Lond B Biol Sci 1979, 205:58I-598.

9. Huxley J: Constant differential growth-ratios and their significance. Nature 1924, I 14:895-896.

10. Huxley J, Teissier G: Terminology of relative growth rates. Nature 1936, I37:780-78|.

II. Carroll SB: Endless Forms Most Beautiful: The New Science of Evo Devo. New York: WW Norton; 2006.

12. Gerhart J, Kirschner M: Cells, Embryos, and Evolution. Oxford: Blackwell Publishers; 1997.

13. Raff RA: The Shape of Life. Chicago: University of Chicago Press; 1996.

14. Stern DL, Emlen DJ: The developmental basis for allometry in insects. Development 1999, 126:1091-1I0I.

15. Abzhanov A, Kuo WP, Hartmann C, Grant BR, Grant PR, Tabin $\mathrm{CJ}$ : The calmodulin pathway and evolution of elongated beak morphology in Darwin's finches. Nature 2006, 442:563-567.

16. Abzhanov A, Protas M, Grant BR, Grant PR, Tabin CJ: Bmp4 and morphological variation of beaks in Darwin's finches. Science 2004, 305: | $462-\mid 465$.

17. Aczel J, Dhombres J: Functional Equations in Several Variables. Cambridge: Cambridge University Press; 1989.

18. Barrenblatt GI: Scaling, Self-similarity, and Intermediate Asymptotics. Cambridge: Cambridge University Press; 1996.

19. Striedter GF: Principles of Brain Evolution. Sunderland, MA: Sinauer; 2005.

20. West GB, Brown JH, Enquist BJ: The fourth dimension of life: fractal geometry and allometric scaling of organisms. Science 1999, 284:1677-1679.

21. Stevens CF: An evolutionary scaling law for the primate visual system and its basis in cortical function. Nature 200 I, 4I I: 193- 195.

22. Lee S, Stevens CF: General design principle for scalable neural circuits in a vertebrate retina. Proc Natl Acad Sci USA 2007, 104: I293 I- 12935.

23. Darwin CR: Journal of Researches into the Natural History and Geology of the Countries Visited During the Voyage of H.M.S. Beagle Round the World, Under the Command of Capt. Fitz Roy, R.N. 2d edition. London: John Murray; 1845. 